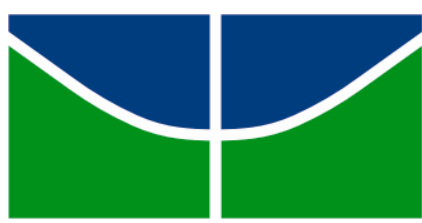

Universidade de Brasília

Instituto de Ciências Biológicas

Programa de Pós-Graduação em Zoologia

Diversidade de Cetoniidae (Insecta: Coleoptera) no Cerrado do Distrito Federal

Juliane Evangelista Neto 

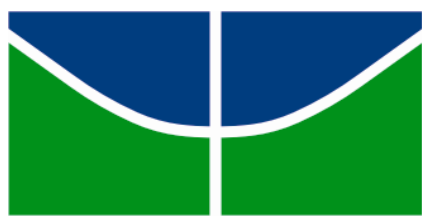

Universidade de Brasília

Instituto de Ciências Biológicas

Programa de Pós-Graduação em Zoologia

\title{
Diversidade de Cetoniidae (Insecta: Coleoptera) no Cerrado do Distrito Federal
}

\author{
Juliane Evangelista Neto
}

Dissertação apresentada ao Programa de Pós-Graduação em Zoologia como um dos requisitos para a obtenção do título de Mestre em Zoologia.

Orientadora: Profa. Dra. Marina Regina Frizzas

Brasília, 2015 
Dissertação de Mestrado

\section{JULIANE EVANGELISTA NETO}

Título:

"Diversidade de Cetoniidae (Insecta: Coleoptera) no Cerrado do Distrito Federal"

\section{Banca Examinadora:}

$$
\begin{gathered}
\text { Warina fizzan } \\
\begin{array}{c}
\text { Profa. Dra. Marina Regina Frizas } \\
\text { Presidente } / \text { Orientadora } \\
\text { zoO/UnB }
\end{array}
\end{gathered}
$$

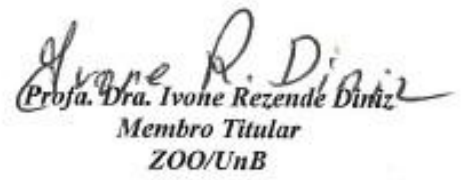
ZOO/UnB

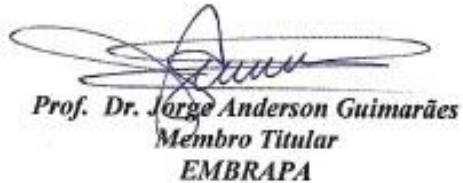

EMBRAPA

Prof. Dr. Raul Alberto Laumann

Membro Suplente

EMBRAPA

Em 31 de março de 2015. 


\section{AGRADECIMENTOS}

Aos meus pais, Douglas e Sônia, que não mediram esforços para que eu chegasse até esta etapa de minha vida.

Á Professora Marina Regina Frizzas pela oportunidade para o desenvolvimento deste estudo e orientação.

Ao Dr. Lee Dyer (Universidade de Nevada, Reno) e André Rangel por auxiliarem com os dados estatísticos.

Ao Dr. Fernando Z. Vaz de Mello (UFMT, Cuiabá) por auxiliar com as identificações dos Cetoniidae.

Ao Thiago, meu irmão querido e Dauglio, tio amado, por me auxiliarem com as fotos dos espécimes.

Ao Bê por constante incentivo e apoio nas horas difíceis.

Aos amigos e companheiros de laboratório, em especial Amanda Coelho, João Silva, Marcus Celani, Thiara Bernardes, Túlio Campos e Maycon Aquino que me auxiliaram nas inúmeras idas ao campo e fizeram companhia durante toda essa jornada.

Á Universidade de Brasília e ao Programa de Pós-Graduação em Zoologia. 


\section{SUMÁRIO}

ÍNDICE DE FIGURAS ..................................................................................... iv

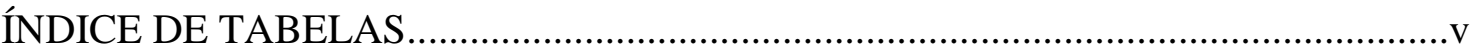

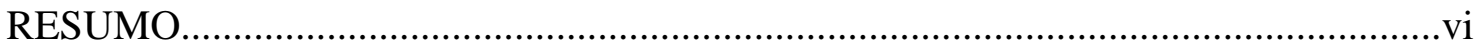

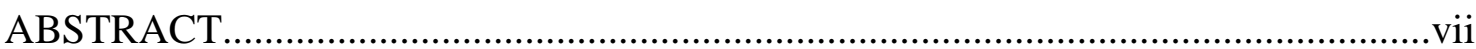

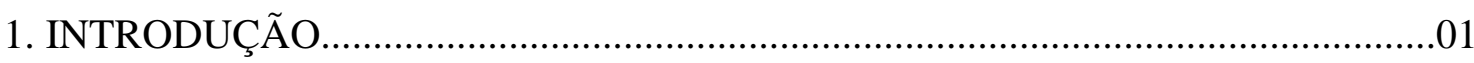

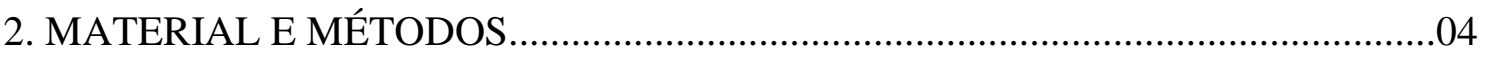

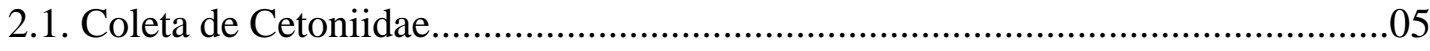

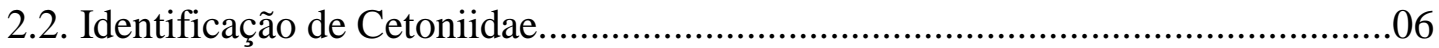

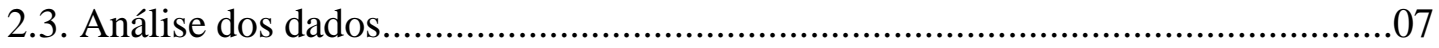

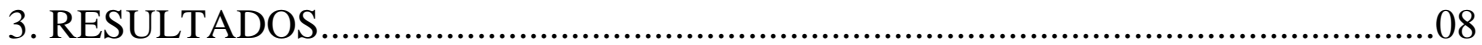

4. DISCUSS ÃO

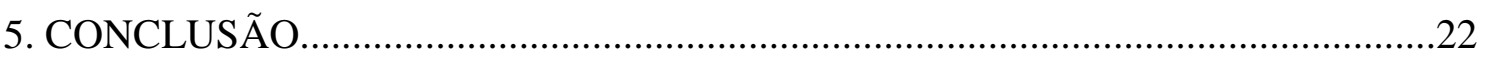

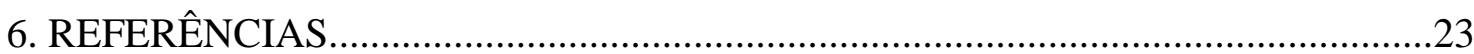




\section{ÍNDICE DE FIGURAS}

Figura 1. Fazenda Água Limpa (FAL) demarcada em vermelho e área de coleta de Cetoniidae indicada pelo ponto vermelho 4

Figura 2. a) Modelo da armadilha utilizada para a coleta de Cetoniidae no campo. b) Armadilha iscada instalada em campo na altura de 1,50 $\mathrm{m}$ do nível do solo. Coleta realizada na FAL no período de outubro de 2013 a setembro de 2014 .5

Figura 3. Esquema da distribuição das armadilhas em campo para a coleta de Cetoniidae na FAL no período de outubro de 2013 a setembro de 2014. A isca de abacaxi e B isca de banana 6

Figura 4. Espécies de Cetoniidae coletadas na FAL em armadilhas iscadas com frutas fermentadas no período de outubro de 2013 a setembro de 2014. (a) Allorrhina baeri; (b) Allorrhina cornifrons, macho (b) e fêmea (b'); (c) Euphoria lurida; (d) Gymnetis flavomarginata; (e) Gymnetis hebraica; (f) Gymnetis rubrocincta, (g) Gymnetis rufilatris; (h). Gymnetis sp.; (i) Allorrhina cincta; (j) Hologymnetis undulata, (k) Hoplopyga albiventris; (1) Hoplopyga brasiliensis; (m) Hoplopyga liturata; (n) Hoplopygathrix atropurpurea, macho (n) e fêmea (n'); (o) Inca bonplandi, macho (o) e fêmea (o'); (p) Marmarina maculosa. Barra lateral: 10,0 mm

Figura 5. Análise circular para riqueza e abundância de Cetoniidae coletados na FAL em armadilhas iscadas com frutas fermentadas no período de outubro de 2013 a setembro de 2014. $X=$ Uniformidade de Rayleigh, $* *=p<0,0001$

Figura 6. Relação mensal do total de Cetoniidae coletado na FAL em armadilhas iscadas com frutas fermentadas no período de outubro de 2013 a setembro de 2014 com as variáveis climáticas

Figura 7. Correlação com previsão de elipse em 95\% para a índice de simpson com as variáveis climáticas. Coleta de Cetoniidae realizada na FAL em armadilhas iscadas com frutas fermentadas no período de outubro de 2013 a setembro de 


\section{ÍNDICE DE TABELAS}

Tabela 1 - Espécies e abundância de Cetoniidae coletadas na FAL em armadilhas iscadas com banana e abacaxi em área de cerrado sensu stricto no período de outubro de 2013 a setembro de 2014 em Brasília, DF.

Tabela 2 - Total de indivíduos coletados por espécie de Cetoniidae na FAL em armadilha iscada com fruta fermentada no período de outubro de 2013 a setembro de 2014 12

Tabela 3 - Teste de ANCOVA para correlação entre a diversidade de Cetoniidae (índice de Simpson) coletados na FAL em armadilha iscada com fruta fermentada no período de outubro de 2013 a setembro de 2014 e as variáveis climáticas. .16 


\section{RESUMO}

A família Cetoniidae é um grupo diverso que possui cerca de 4.000 espécies. No Brasil são registradas 72 espécies e 24 gêneros. Pouco se sabe sobre a família na região Central do Brasil e, apesar da importância ecológica e agrícola desta família, não existem trabalhos realizados no Cerrado do Distrito Federal. Nesse sentido, o objetivo deste trabalho foi avaliar a diversidade e a variação temporal de Cetoniidae em área de Cerrado no Distrito Federal e verificar se a abundância e riqueza de espécies são influenciadas pelas variáveis climáticas. O trabalho foi realizado em uma área de Cerrado sensu stricto na Fazenda Água Limpa (FAL) em Brasília, Distrito Federal. A coleta de adultos de Cetoniidae foi realizada semanalmente usando 40 armadilhas iscadas com dois tipos de iscas (banana e abacaxi) que ficaram em campo por 12 meses ininterruptos (outubro de 2013 a setembro de 2014). As armadilhas foram instaladas cerca de 1,50 m do nível do solo, distribuídas em quatro transectos de $80 \mathrm{~m}$ e espaçadas $20 \mathrm{~m}$ entre si. Foram coletados 398 indivíduos, 9 gêneros e 16 espécies. O Cerrado do Distrito Federal abriga 22,2\% do total de espécies registradas no Brasil. As principais espécies coletadas foram Gymnetis rufilatris com 34,4\%, Gymnetis hebraica com 33,2\% e Inca bonplandi com 13,1\% do total coletado. O maior número de indivíduos e de espécies coletadas ocorre logo após as primeiras chuvas, principalmente no mês de novembro. A variação temporal foi indicada através do teste de uniformidade de Rayleigh que acompanha a sazonalidade do Cerrado, com maior número de indivíduos e de espécies no período chuvoso. A temperatura e umidade são os fatores climáticos que influenciaram a diversidade de Cetoniidae. Este foi o primeiro trabalho realizado com foco na família Cetoniidae no Cerrado sensu stricto do Distrito Federal com registro das espécies existentes.

Palavras-chave: armadilha iscada, biodiversidade, besouro de flor, Cetoniinae, frutas fermentadas. 


\section{ABSTRACT}

The Cetoniidae family is a diverse group that has about 4,000 species. In Brazil are reported 72 species and 24 genera. Little is known about the family in the Central region of Brazil and, despite the ecological and agricultural importance of this family, there are no studies was conducted in the Cerrado of the Distrito Federal. In this sense, the aim of this study was to survey the diversity and temporal variation Cetoniidae in Cerrado biome in the Distrito Federal and verify if climate variables influence the abundance and species richness of Cetoniidae. The study was conducted in an area of Cerrado sensu stricto in Fazenda Água Limpa (FAL) in Brasília, Distrito Federal. The collection of Cetoniidae adults were conducted weekly using 40 traps baited with two types of bait (banana and pineapple) that remained on the field for 12 consecutive months (October 2013 to September 2014). The traps were installed about $1.50 \mathrm{~m}$ from ground level, divided into four transects of $80 \mathrm{~m}$ spaced $20 \mathrm{~m}$ apart for each type of bait. Were collected 398 specimens, 16 species in 9 genera. The Cerrado biome of Distrito Federal has $22.2 \%$ of the total species reported in Brazil. The main species were Gymnetis rufilatris with $34.4 \%$, Gymnetis hebraica with $33.2 \%$ followed by Inca bonplandi with $13.1 \%$ of the collection. The largest number of individuals and species sampled occurs soon after the first rains, especially in November. Temporal variation was confirmed by the Rayleigh test of uniformity following the seasonality of the Cerrado, with the largest number of individuals and species in the rainy season. The temperature and humidity are the climatic factors that influence the diversity of Cetoniidae. This was the first work done with the focus on Cetoniidae family in the Cerrado sensu stricto of the Distrito Federal for the purpose of record the existing species.

Key-words: baited trap, biodiversity, flower beetle, Cetoniinae, fermented fruit. 


\section{INTRODUÇÃ̃o}

A superfamília Scarabaeoidea (Insecta: Coleoptera) possui aproximadamente 35 mil espécies conhecidas (Ratcliffe \& Paulsen, 2008). Por ser um grupo grande dispõem de adaptações para a maioria dos habitats, apresentando uma alimentação variada como fungívora, necrófaga, coprófaga, saprófaga, fitófaga e algumas carnívoras (Arnett et al., 2002; Ratcliffe, 2002). Os Scarabaeoidea apresentam como características distintas as antenas lameladas (Ritcher, 1958) e pernas anteriores geralmente fossoriais (Costa Lima, 1953). São conhecidos por serem pragas agrícolas (Oliveira \& Frizzas, 2013), decompositores de matéria orgânica (Sánchez-Galván et al., 2014), dispersores de sementes (Andresen \& Feer, 2005), polinizadores (Seymour et al., 2003), predadores (Vaz de Mello et al., 1998), bioindicadores de qualidade ambiental (Halffter \& Favila, 1993), auxiliar no controle biológico (Rodrigues et al., 2013a) e por fazer parte da dieta de pequenos mamíferos.

Apesar de Scarabaeoidea ser considerado um grupo relativamente bem estudado, a sua filogenia continua não resolvida (Micó et al., 2008), por essa razão não existe consenso quanto à divisão de famílias e subfamílias, e o número relativo a esses táxons varia de acordo com o sistema de classificação adotado (Ratcliffe \& Jameson, 2004). De acordo com Cherman \& Morón (2014) a subfamília Cetoniinae da família Melolonthidae deve ser elevada a família por conter características próprias que os determinam e devido a sua recente confirmação como clado monofilético irmão desse agrupamento. Portanto, neste trabalho adotou-se a divisão proposta por Morón (2010) que considera Scarabaeoidea dividida em 12 famílias, sendo elas: Cetoniidae, Geotrupidae, Glaphyridae, Glaresidae, Hybosoridae, Lucanidae, Melolonthidae, Ochodaeidae, Passalidae, Pleocomidae, Scarabaeidae e Trogidae.

A família Cetoniidae é um grupo diverso, que possui cerca de 4.000 espécies, 300 das quais ocorrem no continente Americano (Orozco, 2012), dividida em quatro subfamílias: Cetoniinae, Trichiinae, Valginae e Osmodermatinae (Cherman \& Morón, 2014). Os Cetoniidae possuem caracteres próprios que os delimitam, como clípeo cobrindo labro e mandíbulas, seis esternitos abdominais visíveis, antenas formadas por 10 segmentos cujas inserções são visíveis de cima e uma acentuada marginalização pósumeral que possibilita um vôo rápido e sonoro com os élitros fechados (Cherman \& Morón, 2014). 
No Brasil são relatadas 72 espécies e 24 gêneros (Puker et al., 2014). As larvas de apenas 61 espécies são conhecidas e elas podem ser encontradas no solo, em compostos orgânicos (Pejaver \& Gujarathi, 2013), bolos fecais secos, troncos de árvores em decomposição (Orozco \& Pardo-Locarno, 2004), no tecido de Bromeliaceae (Krell et al., 2002) e em associação com ninhos de formigas e cupins (Puker et al., 2012).

Estudos com Cetoniidae realizados no Brasil abordaram principalmente descrições de estágios imaturos (Vanin \& Costa, 1984; Micó et al., 2000; 2001), algumas revisões e descrições de adultos (Ratcliffe \& Deloya, 1992; Ratcliffe \& Micó, 2001; Ratcliffe, 2005; 2010; Orozco, 2012) e caracterização da diversidade de espécies (Gonçalves \& Louzada, 2005; Puker et al., 2012). A maioria desses estudos ocorreu nas regiões Sul e Sudeste do país, portanto, pouco se sabe sobre a família na região Central do Brasil. Foram realizados apenas dois trabalhos em uma área de transição Cerrado/Pantanal onde Garcia et al. (2013) encontraram três espécies enquanto Rodrigues et al. (2013b) mencionaram nove espécies de Cetoniidae.

Cetoniidae estão distribuídos em todo o mundo, e não são representados apenas por espécies coloridas com brilho metálico, mas também por espécies com texturas de veludo e traços coloridos que geram padrões (Kumbhar et al., 2012). A biologia de Cetoniidae é bastante variável, algumas espécies completam o ciclo em poucos meses, enquanto outras requerem mais de um ano (Micó \& Galante, 2003; Kumbhar et al., 2012; Šípek \& Král, 2012). A maioria dos Cetoniidae adultos apresenta hábitos alimentares diurnos, alimentando-se de néctar, pólen, exsudados de plantas e frutos maduros (Ritcher, 1958; Krikken, 1984a). Adultos de algumas espécies de Cetoniidae são relatados como pragas agrícolas, hortícolas, ornamentais e polinizadores em muitas partes do mundo (Molina, 2001; Vuts et al., 2008; Vuts et al., 2010; Aydin, 2011; Subchev et al., 2011). Adultos de Chiloloba orientalis (Gory \& Percheron) foram relatados se alimentando de inflorescência de milho, sorgo e pastagens sendo que a emergência dos adultos coincide com o período de florescimento das plantas hospedeiras (Kumbhar et al., 2012). Euphoria lurida (Fabricius) causa danos em plantas de milho na fase reprodutiva (Cunha et al., 2007), em frutíferas e plantas ornamentais (Gallo et al., 2002). Na África do Sul as espécies Atrichelaphinis tigrina (Olivier) e Cyrtothyrea marginalis (Swartz) são polinizadores especializados em três espécies da família Apocynaceae (Shuttleworth \& Johnson, 2009). 
Trabalhos de coleta, através de armadilhas iscadas, usualmente empregam diferentes frutos como abacaxi, banana e manga que podem ou não estar em processo de fermentação. Para fermentação, em sua maioria, é utilizado caldo de cana, mas existem trabalhos que utilizam cerveja (Pacheco et al., 2006; Jakl, 2009; Orozco, 2012) podendo assim existir diferenças na abundância e riqueza de espécies coletadas.

Na região Central do Brasil encontra-se o Cerrado com 204,7 milhões de hectares. Este bioma apresenta, em termos de biodiversidade e fitofisionomias, alta heterogeneidade com forte variação temporal. Além de ser o segundo maior bioma do Brasil é considerado um hotspot da biodiversidade que necessita de prioridade para conservação (Myers et al., 2000; Sano et al., 2010). Estima-se que 28 espécies de Scarabaeoidea são endêmicas no bioma (Vaz de Mello, 2000), em compensação apenas $20 \%$ dos 204,7 milhões de hectares estão em seu estado natural, e menos de $2 \%$ estão em áreas de preservação como parques e reservas (Silva et al., 2013).

O Cerrado dispõe de recursos alimentares e condições ambientais para o estabelecimento de alta riqueza de espécies da família Cetoniidae ainda não registrada. Por estar espalhada por todo o globo terrestre, especialmente em florestas tropicais (Krikken, 1984b), ser uma família diversa, apresentando em sua maioria endemismo, surgem os seguintes questionamentos: Quais espécies de Cetoniidae estão presentes no Cerrado do Distrito Federal? A diversidade de Cetoniidae é influenciada pelas variações sazonais que ocorrem na região do Distrito Federal?

Devido ao aumento da agricultura e da agropecuária no Cerrado (Brannstrom et al., 2008), da existência de poucas áreas de preservação, do baixo número de espécies de Cetoniidae descritas no Cerrado em relação a outros biomas, do potencial do bioma em abrigar espécies do grupo, da importância ecológica e agrícola da família, e por não existir, até o momento, nenhum trabalho realizado no Cerrado do Distrito Federal com Cetoniidae, surge a necessidade de estudos sobre a biodiversidade da família, bem como a demanda de conhecimento da fauna regional para a conservação do grupo e do bioma Cerrado.

Portanto este trabalho tem como objetivos avaliar a diversidade e variação temporal de Cetoniidae em área de Cerrado no Distrito Federal e verificar se a abundância e riqueza de espécies de Cetoniidae são influenciadas pelas variáveis climáticas. 


\section{MATERIAL E MÉTODOS}

O estudo foi realizado na Fazenda Água Limpa (FAL) em Brasília, Distrito Federal em uma área de 1,1 ha de Cerrado sensu stricto $\left(15^{\circ} 57^{\prime 2} 24.38^{\prime \prime S}, 47^{\circ} 56^{\prime} 42.86^{\prime \prime} \mathrm{W}\right.$ e $1.096 \mathrm{~m}$ ), caracterizada pela presença de uma camada descontínua de gramíneas e árvores baixas, inclinadas, tortuosas, com ramificações irregulares que podem ter de 1,5 a 5 metros. A área de coleta localiza-se dentro da área de Primatologia da Fazenda Água Limpa pertencente à Universidade de Brasília - UnB, possui uma área de 4.500 hectares e faz parte da Área de Proteção Ambiental - APA das Bacias do Gama e Cabeça do Veado e tem, no seu interior, a Área Relevante de Interesse Ecológico - ARIE Capetinga/Taquara, também denominada Estação Ecológica da Universidade de Brasília (Figura 1).

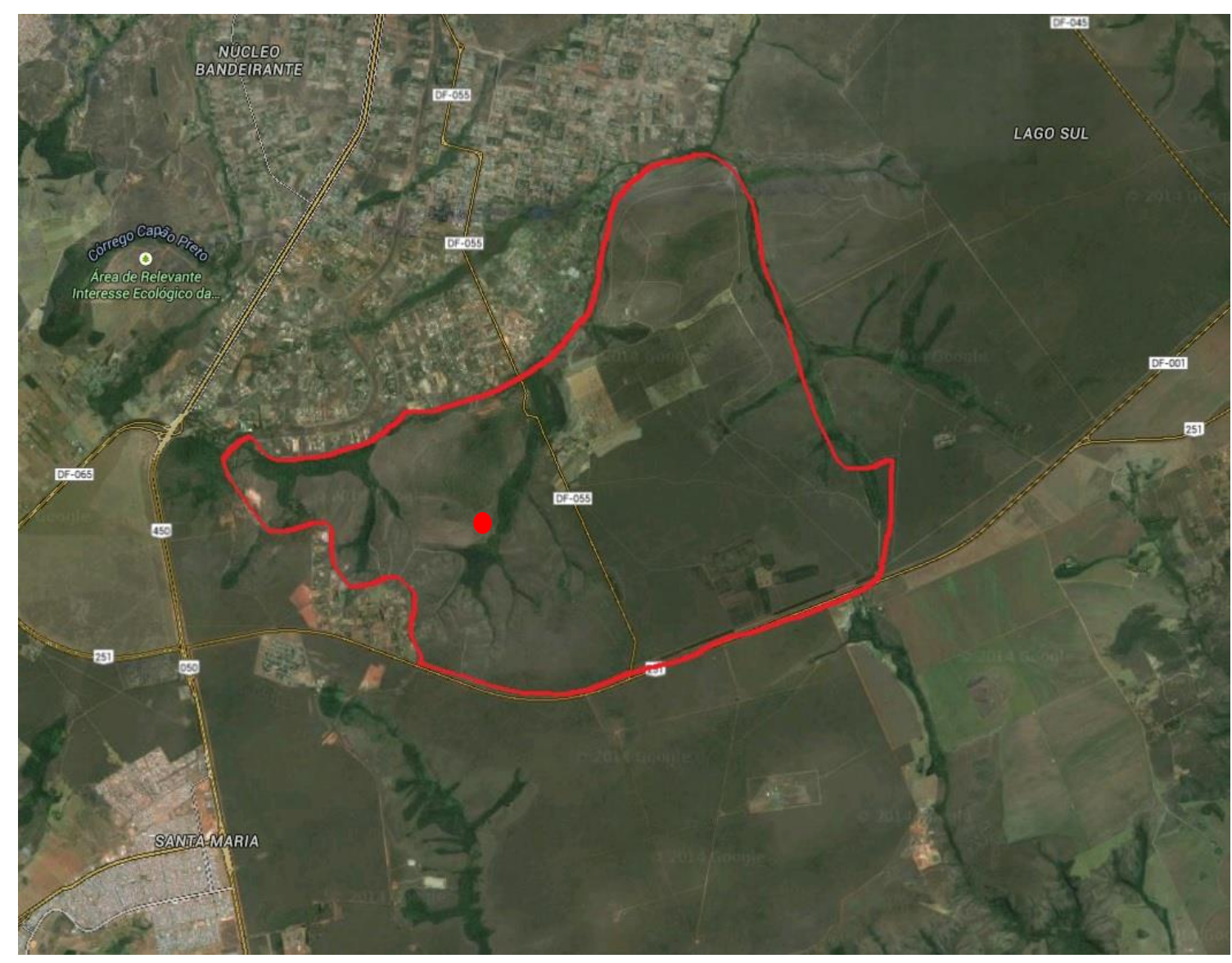

Figura 1. Fazenda Água Limpa (FAL) demarcada em vermelho e área de coleta de Cetoniidae indicada pelo ponto vermelho. 


\subsection{Coleta de Cetoniidae}

A coleta de adultos de Cetoniidae foi realizada semanalmente por 12 meses, outubro de 2013 a setembro de 2014, por meio de 40 armadilhas iscadas que permaneceram ininterruptamente no campo.

A armadilha consistia de uma garrafa plástica de 2 L com três janelas laterais de $8 \times 8 \mathrm{~cm}$, localizadas $10 \mathrm{~cm}$ acima da base (Figura 2a). Abaixo das janelas foram feitos orifícios laterais para escoamento da água. $\mathrm{Na}$ parte interna das armadilhas foi acondicionado cerca de $150 \mathrm{ml}$ de isca, composta por banana e caldo de cana em 20 armadilhas e nas outras 20, abacaxi e caldo de cana. As iscas fermentaram por 48 horas antes de serem colocadas nas armadilhas. Foram preparadas 80 armadilhas, 40 ficavam no campo, e as outras 40 no laboratório para a troca semanal da isca. As armadilhas foram instaladas cerca de 1,50 m do nível do solo (Figura $2 \mathrm{~b}$ ), distribuídas em quatro transectos de $80 \mathrm{~m}$ e espaçadas $20 \mathrm{~m}$ entre si (Figura 3). Foi estabelecida uma margem de $20 \mathrm{~m}$ do ponto de entrada até a instalação da primeira armadilha para evitar o efeito de borda.
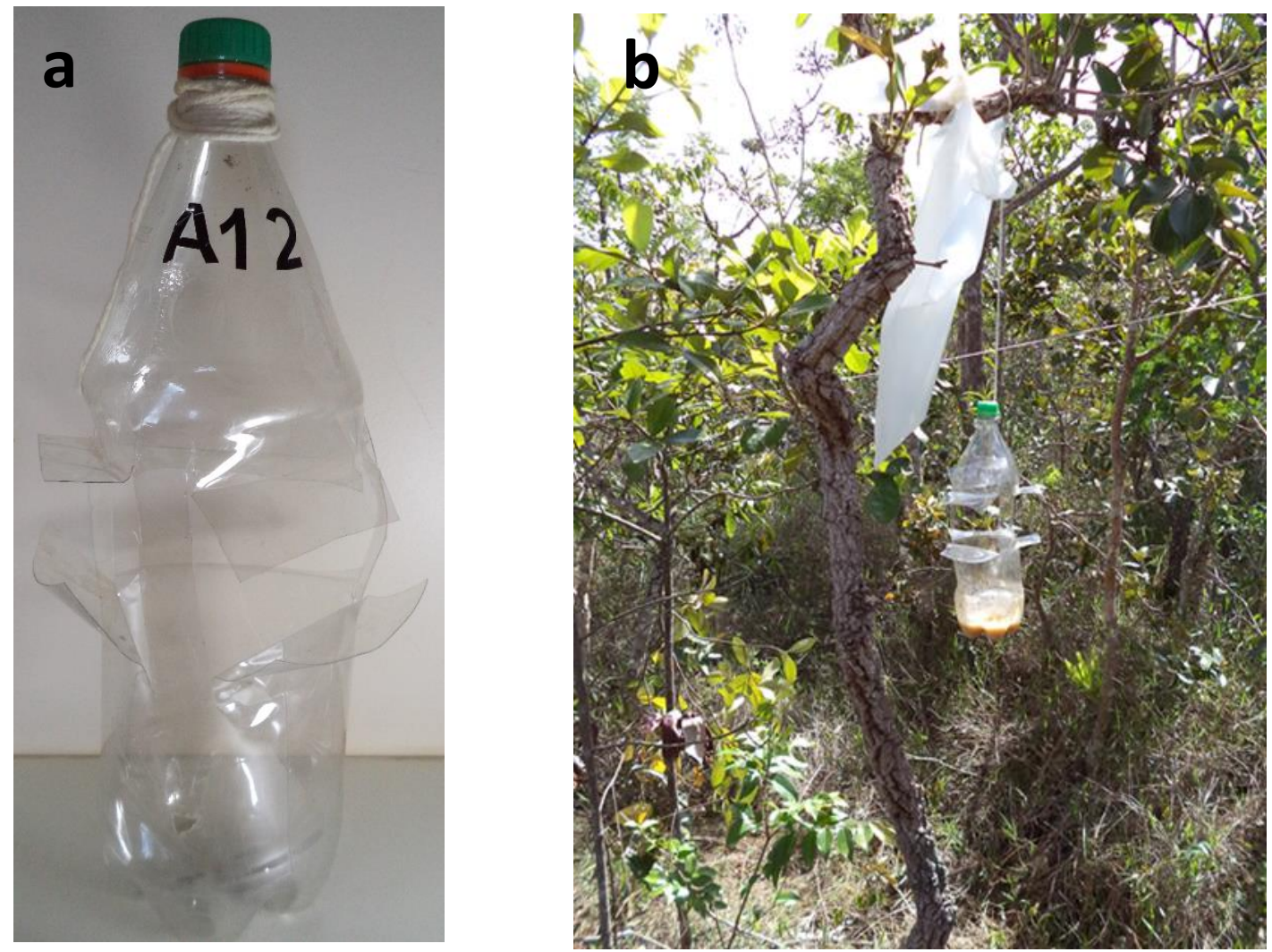

Figura 2. a) Modelo da armadilha utilizada para a coleta de Cetoniidae no campo. b) Armadilha iscada instalada em campo na altura de $1,50 \mathrm{~m}$ do nível do solo. Coleta realizada na FAL no período de outubro de 2013 a setembro de 2014. 


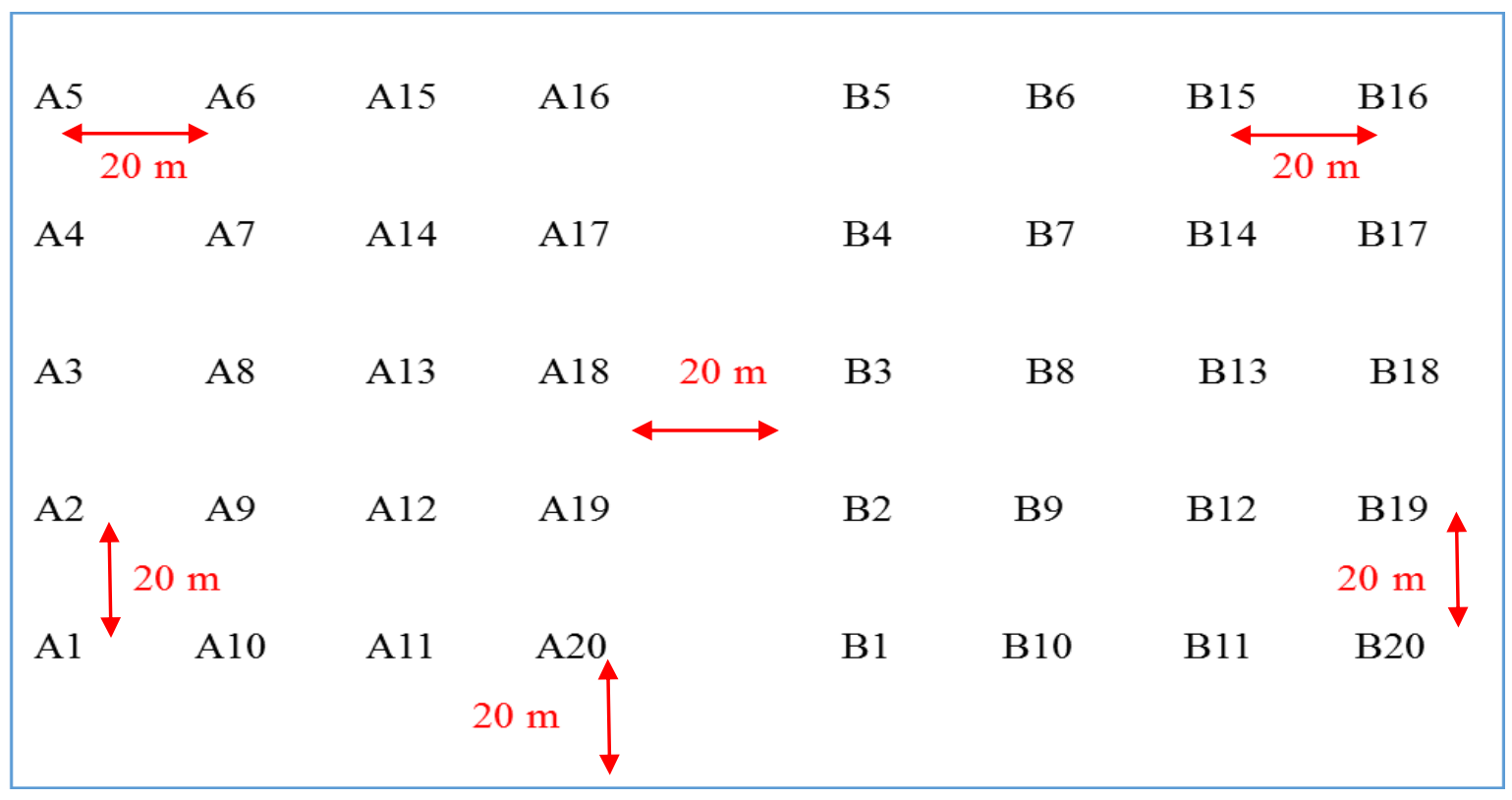

Figura 3. Esquema da distribuição das armadilhas em campo para a coleta de Cetoniidae na FAL no período de outubro de 2013 a setembro de 2014. A isca de abacaxi e B isca de banana.

\subsection{Identificação de Cetoniidae}

Os insetos coletados foram levados ao Laboratório de Entomologia da Universidade de Brasília onde foi realizada a triagem. Todos os Cetoniidae coletados foram montados, etiquetados e, posteriormente realizou-se a separação por espécie e contagem do número de indivíduos por espécie, data de coleta e tipo de isca utilizada. Um exemplar de cada espécie, macho e fêmea quando possível, foi fotografado na posição dorsal por uma câmera Sony DSC-W710.

Os Cetoniidae coletados foram identificados até o menor nível taxonômico possível com o auxílio do especialista Dr. Fernando Zagury Vaz de Mello (Departamento de Biologia e Zoologia do Instituto de Biociências da Universidade Federal de Mato Grosso) por meio de chaves de identificação, por comparação com exemplares existentes na coleção entomológica do Departamento de Zoologia, Instituto de Ciências Biológicas da Universidade de Brasília, Brasília, DF e da coleção do Departamento de Biologia e Zoologia do Instituto de Biociências da Universidade Federal do Mato Grosso, Cuiabá, MT e por dissecação de genitália masculina quando necessário. Para o exame da genitália masculina amoleceram-se os tecidos internos com água quente e sob microscópio 
estereoscópico a peça genital foi exposta com auxílio de pinça, permanecendo presa ao final do abdome. A identificação ocorreu através de comparação.

Vouchers do material coletado estão depositados nas coleções Entomológicas do Departamento de Zoologia da Universidade de Brasília e do Departamento de Biologia e Zoologia da Universidade Federal do Mato Grosso.

\subsection{Análise dos dados}

Os dados climáticos de temperatura, umidade e precipitação foram obtidos por meio do Boletim AgroClima fornecido pela Faculdade de Agronomia e Medicina Veterinária (FAV/UnB) que mantém na Fazenda Água Limpa uma Área Experimental de Agroclimatologia.

O desenho experimental apresenta pseudo-replicatas portanto, para analisar os dados referentes à variação temporal foi realizado o teste de Uniformidade de Rayleigh que analisa a relação funcional de variáveis sob um conceito de homogeneidade dimensional (Mendoza, 1993), já em relação à abundância e riqueza de espécies para os meses foi utilizada a análise circular (Agostinelli \& Lund, 2013). Para verificar se a abundância e riqueza de espécies de Cetoniidae são influenciadas pelas variáveis climáticas (temperatura, umidade e precipitação) foi utilizada análise de Covariância ANCOVA, fruta fermentada como variável independente, a diversidade de Simpson como variável resposta e as variáveis climáticas como co-variáveis.

As análises e testes foram desenvolvidos por meio do software R Core Team ( $\mathrm{R}$ Core Team, 2014) e Oriana (Kovach, 2011). 


\section{RESULTADOS}

Foram coletados 398 espécimes, 9 gêneros e 16 espécies de Cetoniidae (Tabela 1 e Figura 4) em 50 coletas realizadas no período de amostragem. As espécies mais abundantes foram Gymnetis rufilatris (Illiger) com 34,4\%, Gymnetis hebraica (Drapiez) com 33,2\% e Inca bonplandi (Gyllenhall) com 13,1\% do total coletado.

$\mathrm{Na}$ isca de abacaxi coletou-se 53,5\% dos espécimes e 13 espécies e na isca de banana 46,5\% dos espécimes e 14 espécies (tabela 1), sendo que 11 espécies $(68,8 \%$ ) foram similares aos dois tipos de iscas. A espécie mais abundante na isca de banana foi G. rufilatris (72 indivíduos) e, G. hebraica (89 indivíduos) na isca de abacaxi, já $I$. bonplandi foi igualmente coletada nas duas iscas (26 indivíduos em cada isca). As espécies Allorrhina baeri Bourgoin, Gymnetis sp. e Hologymnetis undulata (Vigors) foram coletadas apenas na isca de banana e as espécies Gymnetis rubrocincta (Schürhoff) e Marmarina maculosa (Olivier) apenas na isca de abacaxi.

Os Cetoniidae são robustos e possuem o corpo ligeiramente plano, variaram de 1 a $3 \mathrm{~cm}$ de comprimento, possuíam em sua maioria cores sóbrias como marrom, bege, preto e verde musgo mostrando um possível padrão de coloração, no entanto, algumas espécies apresentaram coloração vermelho e amarelo que em contraste com o preto formavam desenhos padronizados (Figura 4). Algumas espécies possuíam padrões de traços finos e profundos (formando sulcos). A textura dos élitros variou de lisa a aveludada (pilosidade). A distinção entre macho e fêmea pode ser feita, em alguns casos, através da presença ou ausência de chifres ou até mesmo da diferença de textura dos élitros, em outros casos existe a necessidade de se observar a parte ventral ou através da dissecação da genitália.

Na tabela 2 está listado o total de indivíduos coletado por espécie no período de doze meses. No período chuvoso ocorreram as coletas com o maior número de indivíduos $(88,9 \%)$ e de espécies $(87,5 \%)$. Dentre os meses do período chuvoso, o mês de novembro representou $48,3 \%$ do total coletado. Já o período de seca representou apenas $11,1 \%$ do total coletado e 5 espécies, sendo que agosto foi o mês com a menor coleta, com apenas 1 indivíduo coletado. Com exceção de G. hebraica, que foi coletada no decorrer de todo o ano, as demais espécies se concentraram no período chuvoso (Tabela 2). Apesar do mês de abril estar no período da seca esse mês representa o momento de transição das estações, foram coletados 31 indivíduos e 5 espécies. 
Tabela 1 - Espécies e abundância de Cetoniidae coletadas na FAL em armadilhas iscadas com banana e abacaxi em área de cerrado sensu stricto no período de outubro de 2013 a setembro de 2014 em Brasília, DF.

\begin{tabular}{|c|c|c|c|}
\hline Espécies & Abacaxi & Banana & Total \\
\hline Allorrhina baeri Bourgoin & - & 1 & 1 \\
\hline Allorrhina cincta (Gory \& Percheron) & 1 & 2 & 3 \\
\hline Allorrhina cornifrons Gory \& Percheron & 2 & 1 & 3 \\
\hline Euphoria lurida (Fabricius) & 9 & 12 & 21 \\
\hline Gymnetis flavomarginata Blanchard & 1 & 4 & 5 \\
\hline Gymnetis hebraica (Drapiez) & 89 & 43 & 132 \\
\hline Gymnetis rubrocincta (Schürhoff) & 3 & - & 3 \\
\hline Gymnetis rufilatris (Illiger) & 65 & 72 & 137 \\
\hline Gymnetis sp. & - & 1 & 1 \\
\hline Hologymnetis undulata (Vigors) & - & 2 & 2 \\
\hline Hoplopyga albiventris (Gory \& Percheron) & 1 & 2 & 3 \\
\hline Hoplopyga brasiliensis (Gory \& Percheron) & 12 & 8 & 20 \\
\hline Hoplopyga liturata (Olivier) & 1 & 5 & 6 \\
\hline Hoplopygothrix atropurpurea (Schaum) & 2 & 6 & 8 \\
\hline Inca bonplandi (Gyllenhall) & 26 & 26 & 52 \\
\hline Marmarina maculosa (Olivier) & 1 & - & 1 \\
\hline Total & 213 & 185 & 398 \\
\hline Número de espécies & 13 & 14 & 16 \\
\hline
\end{tabular}




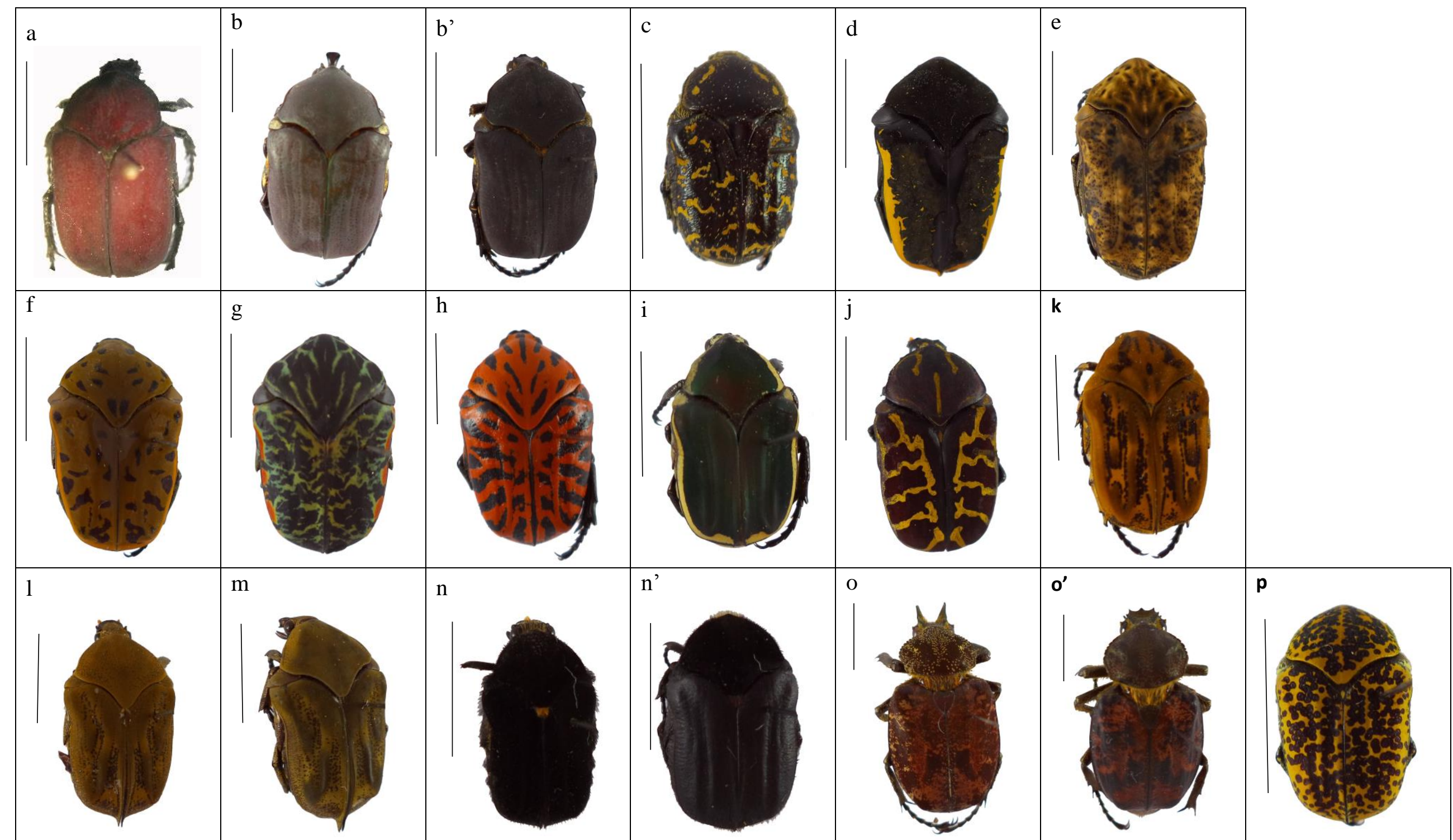

Figura 4. Espécies de Cetoniidae coletadas na FAL no período de outubro de 2013 a setembro de 2014, com isca de fruta fermentada. (a) Allorrhina baeri; (b) Allorrhina cornifrons, macho (b) e fêmea (b'); (c) Euphoria lurida; (d) Gymnetis flavomarginata; (e) Gymnetis hebraica; (f) Gymnetis rubrocincta, (g) Gymnetis rufilatris; (h). Gymnetis sp.; (i) Allorrhina cincta; (j) Hologymnetis undulata, (k) Hoplopyga albiventris; (l) Hoplopyga brasiliensis; (m) Hoplopyga liturata; (n) Hoplopygathrix atropurpurea, macho (n) e fêmea (n'); (o) Inca bonplandi, macho (o) e fêmea (o'); (p) Marmarina maculosa. Barra lateral: 10,0 mm. 
Tabela 2 - Total de indivíduos coletados por espécie de Cetoniidae na FAL em armadilha iscada com fruta fermentada no período de outubro de 2013 a setembro de 2014 .

\begin{tabular}{|c|c|c|c|c|c|c|c|c|c|c|c|c|c|}
\hline \multirow[b]{2}{*}{ Espécie } & \multicolumn{6}{|c|}{ Período chuvoso } & \multicolumn{7}{|c|}{ Período seco } \\
\hline & Out. & Nov. & Dez. & Jan. & Fev. & Mar. & Abr. & Mai. & Jun. & Jul. & Ago. & Set. & Total \\
\hline Allorrhina baeri & & 1 & & & & & & & & & & & 1 \\
\hline Allorrhina cornifrons & 2 & & & 1 & & & & & & & & & 3 \\
\hline Euphoria lurida & 1 & 1 & 10 & 8 & 1 & & & & & & & & 21 \\
\hline Gymnetis flavomarginata & & 1 & & & 1 & 1 & 1 & 1 & & & & & 5 \\
\hline Gymnetis hebraica & 9 & 41 & 30 & 6 & 4 & 9 & 22 & 2 & 2 & 4 & 1 & 2 & 132 \\
\hline Gymnetis rubrocincta & & 1 & 1 & & 1 & & & & & & & & 3 \\
\hline Gymnetis rufilatris & 22 & 88 & 13 & 3 & 3 & 1 & 6 & 1 & & & & & 137 \\
\hline Gymnetis sp. & & 1 & & & & & & & & & & & 1 \\
\hline Heterocotinis cinta & 2 & 1 & & & & & & & & & & & 3 \\
\hline Hologymnetis undulata & & 2 & & & & & & & & & & & 2 \\
\hline Hoplopyga albiventris & 1 & 1 & & & & 1 & & & & & & & 3 \\
\hline Hoplopyga brasiliensis & & 8 & 6 & 4 & 1 & 1 & 1 & & & & & & 21 \\
\hline Hoplopyga liturata & 2 & 1 & & 1 & & & 1 & & & & & & 5 \\
\hline Hoplopygathrix atropurpurea & 3 & 4 & 1 & & & & & & & & & & 8 \\
\hline Inca bonplandi & & 20 & 30 & 2 & & & & & & & & & 52 \\
\hline Marmarina maculosa & 1 & & & & & & & & & & & & 1 \\
\hline Total & 43 & 171 & 91 & 25 & 11 & 13 & 31 & 4 & 2 & 4 & 1 & 2 & 398 \\
\hline Número de espécies & 9 & 14 & 7 & 7 & 6 & 5 & 5 & 3 & 1 & 1 & 1 & 1 & 16 \\
\hline
\end{tabular}


Por meio da análise circular é possível notar a semelhança na riqueza e abundância dos Cetoniidae coletados (Figura 5). O pico de abundância e de riqueza dos Cetoniidae ocorreu no mês de novembro $\left(290^{\circ}\right)$. A abundância de Cetoniidae mostrou um pico bem definido no mês de novembro, já para a riqueza a distribuição é mais uniforme ao longo do ano, apesar do pico também ocorrer no mês de novembro (Figura 5). Para testar a ocorrência de variação temporal utilizou-se o teste de Uniformidade de Rayleigh. Houve variação temporal significativa para a abundância e riqueza de espécies de Cetoniidae (X= Uniformidade de Rayleigh, $* *=p<0,0001)$.

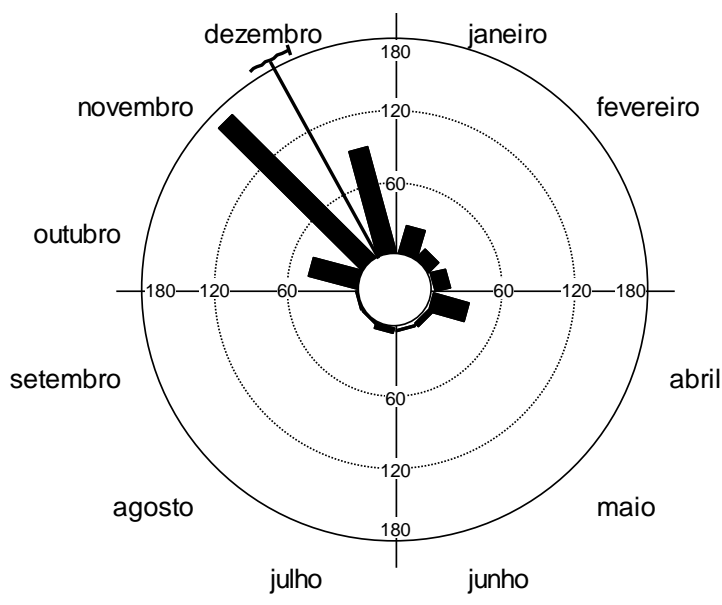

\section{Riqueza}

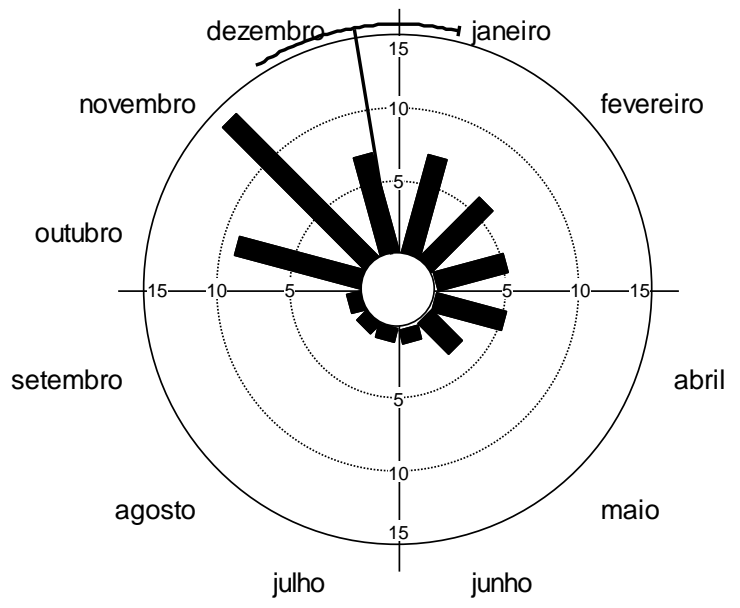

Figura 5. Análise circular para riqueza e abundância de Cetoniidae coletados na FAL em armadilhas iscadas com frutas fermentadas no período de outubro de 2013 a setembro de 2014. $\mathrm{X}=$ Uniformidade de Rayleigh, $* *=\mathrm{p}<0,0001$. 
Observou-se que com o início da chuva, em outubro, houve um aumento na abundância de Cetoniidae coletados, sendo que no mês de novembro em decorrência das primeiras chuvas e revoadas para cópula, a coleta foi ainda maior (Figura 6). O Cerrado apresenta 2 picos de precipitação e 2 picos significativos de abundância para a família Cetoniidae

Assim como para a precipitação, também existiram dois picos de umidade, em dezembro e março. Pode-se observar que no período das coletas a umidade máxima variou entre $75 \%$ e $85 \%$ (Figura 6). A temperatura máxima entre outubro a abril foi de $20,2^{\circ} \mathrm{C}$, meses onde houve as coletas com maior número de indivíduos e a partir de maio existe a queda de temperatura e de indivíduos coletados (Figura 6). 

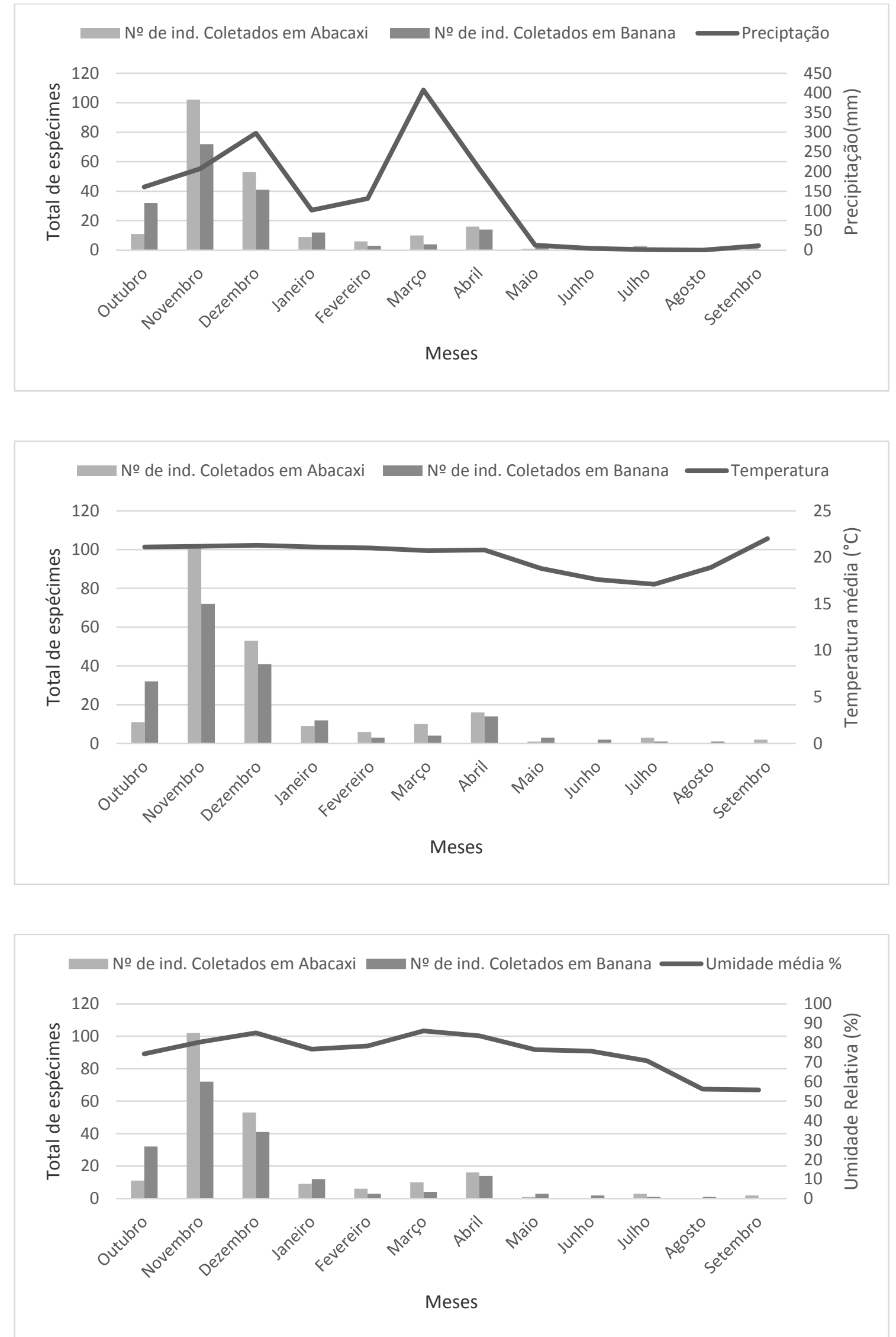

Figura 6. Relação mensal do total de Cetoniidae coletado na FAL em armadilhas iscadas com frutas fermentadas no período de outubro de 2013 a setembro de 2014 com as variáveis climáticas. 
Para averiguar se a abundância e riqueza de espécies de Cetoniidae são influenciadas pelas variáveis climáticas foi realizado o teste de ANCOVA. Pelo teste realizado observou-se que as três variáveis climáticas estão correlacionadas com a diversidade de Cetoniidae (Figura 7), porém, apenas as variáveis temperatura e umidade possuem efeito estatístico significativo na diversidade de Cetoniidae (Tabela 3).

Tabela 3. Teste de ANCOVA para correlação entre a diversidade de Cetoniidae (índice de Simpson) coletados na FAL em armadilha iscada com fruta fermentada no período de outubro de 2013 a setembro de 2014 e as variáveis climáticas.

\begin{tabular}{lcccc}
\hline Variável & G.L. & Média & Valor de F & $p$ \\
\hline Temperatura & 1 & 2,97310353 & 24,94 & $<0,0001$ \\
Precipitação & 1 & 0,00997493 & 0,08 & 0,7725 \\
Umidade & 1 & 2,78754018 & 23,38 & $<0,0001$ \\
\hline
\end{tabular}



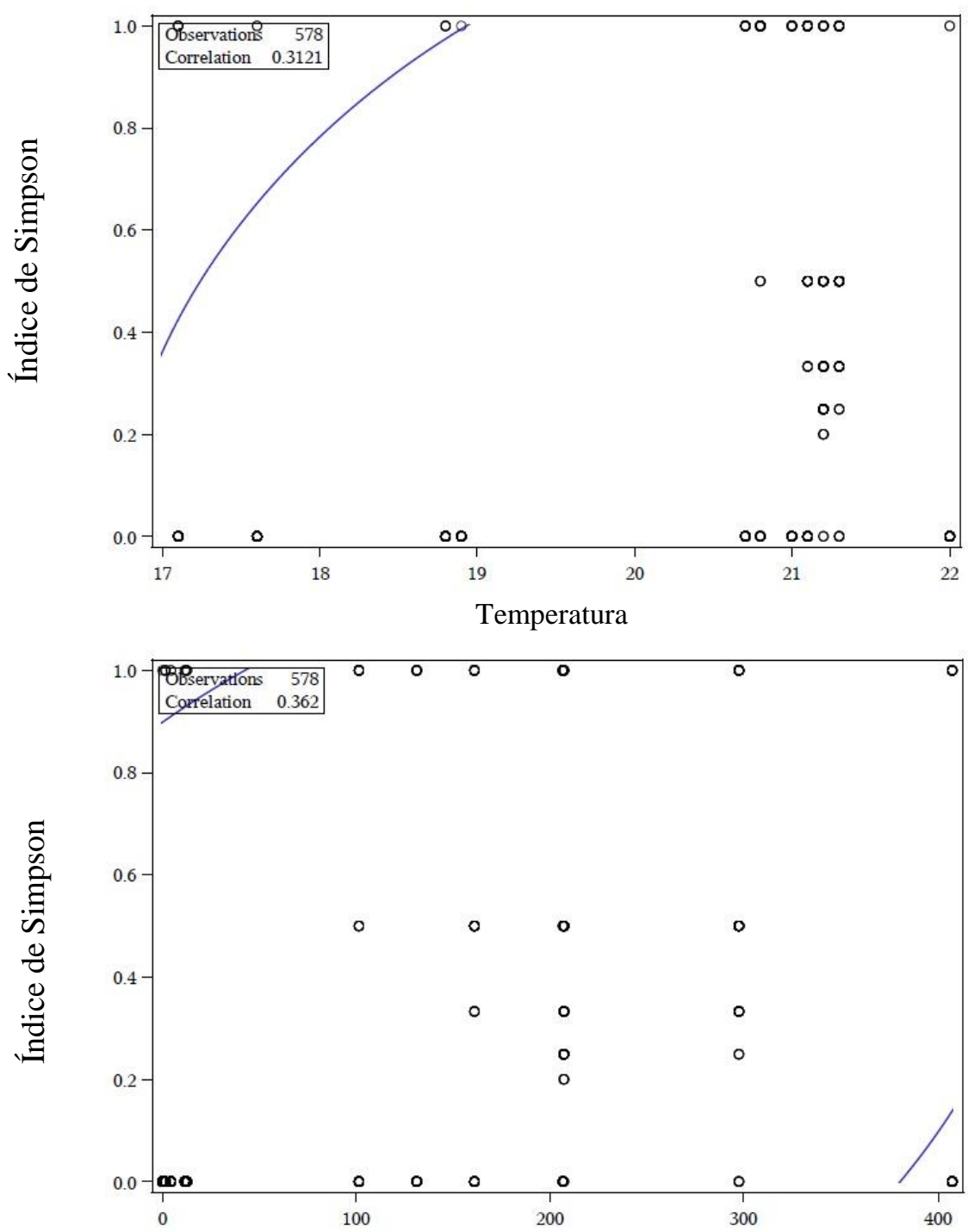

Precipitação

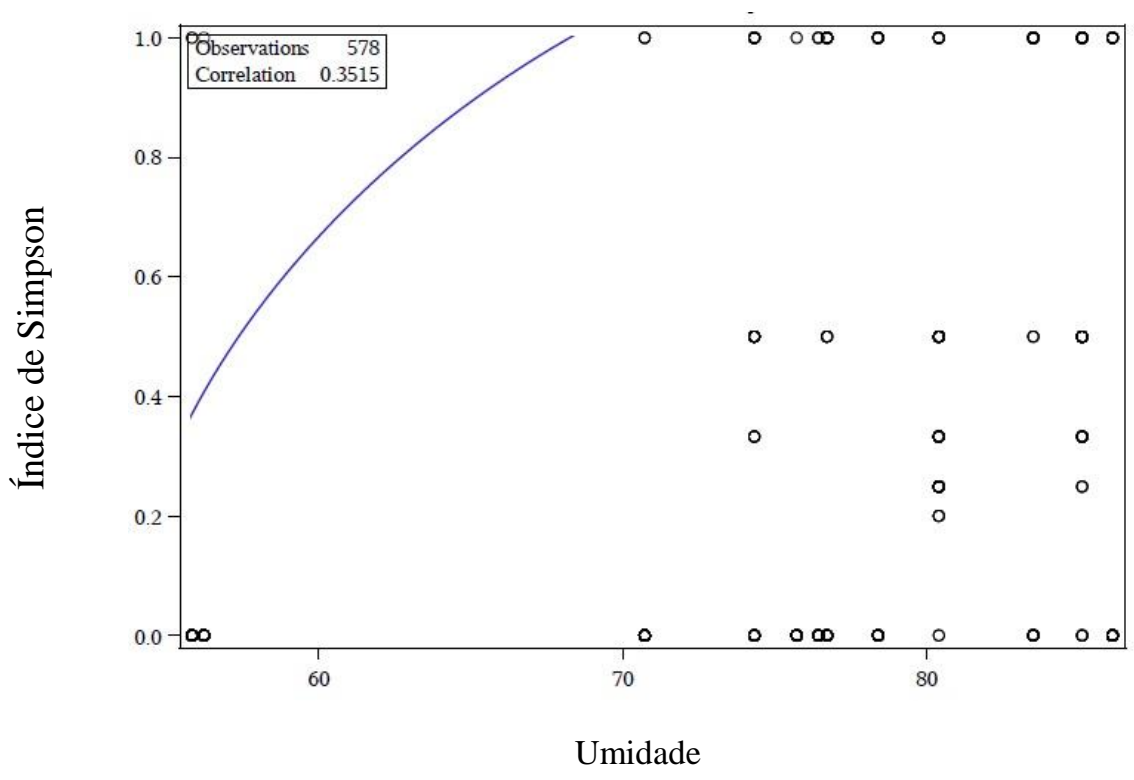

Figura 7. Correlação com previsão de elipse em $95 \%$ para a índice de simpson com as variáveis climáticas. Coleta de Cetoniidae realizada na FAL em armadilhas iscadas com frutas fermentadas no período de outubro de 2013 a setembro de 2014. 


\section{DISCUSSÃO}

O presente estudo procurou caracterizar a diversidade de Cetoniidae e relatar quais os principais gêneros e espécies que ocorrem no cerrado do Distrito Federal, já que a maioria dos estudos realizados até o momento, ocorreu nas regiões Sul e Sudeste do país.

Revisão publicada recentemente reuniu o conhecimento a respeito dos Scarabaeoidea na área de abrangência do Cerrado por meio dos estudos científicos publicados nos últimos 30 anos (1982 - 2012) e mostrou que dos 64 trabalhos recuperados apenas dois tratavam de Cetoniidae, no entanto, nenhum destes dois artigos era específico da família (Luçardo et al., 2014).

Neste estudo foram coletados 398 indivíduos, 16 espécies e 9 gêneros. Para o Brasil há registros de 72 espécies e 24 gêneros (Puker et al., 2014), portanto, o Cerrado sensu stricto do Distrito Federal abriga, pelo menos, 22,2\% das espécies e 37,5\% dos gêneros conhecidos.

Foram realizadas coletas ativas durante um ano de adultos e larvas de Cetoniidae em troncos de árvores em decomposição em uma área de transição de Cerrado e Pantanal em Aquidauana, Mato Grosso do Sul, e encontraram larvas de 3 espécies (Hoplopyga boliviensis (Moser), H. liturata e M. maculosa) e apenas 2 espécies de adultos ( $H$. boliviensis e $H$. liturata) (Garcia et al., 2013). Foram realizadas coletas também com armadilha de fruta (banana e caldo de cana) durante um ano em uma área de transição de Cerrado e Pantanal em Piraputanga, Mato Grosso do Sul, e coletaram 68 indivíduos e nove espécies (Rodrigues et al., 2013b). Apesar de a região possuir um fragmento de Cerrado, em ambas as coletas, a riqueza e abundância são relativamente menores quando comparadas ao Cerrado sensu stricto do Distrito Federal onde foram encontradas 16 espécies e 398 indivíduos.

Das 16 espécies coletadas neste estudo, três ainda não haviam sido relatadas para o Cerrado, são elas: A. cornifrons, G. flavomarginata e G. rubrocincta. Existem relatos, registros e alguns estudos para 11 das 16 espécies encontradas neste estudo (Ratcliffe, 2015; Di Iorio, 2013; Micó et al., 2000; Rodrigues et al., 2013b; Ratcliffe \& Deloya, 1992). Sendo que a espécie mais estudada até o momento é E. lurida principalmente pelo fato de ter sido relatada causando prejuízos em algumas culturas. 
A espécie A. baeri foi registrada apenas para o Brasil na América do Sul (Di Iorio, 2013) com registro de coleta em Goiás (Ratcliffe, 2004). Para A. cornifrons existe apenas um registro em São Paulo (Di Iorio, 2013). Euphoria lurida está presente apenas na América do Sul (Argentina, Bolívia, Brasil, Paraguai e Uruguai). Possui distribuição anual, embora no Cerrado tenha sido coletada apenas na época de chuva. No Brasil foi registrada no Distrito Federal, Minas Gerais, Pará, Paraná, Rio de Janeiro, Rio Grande do Sul, Santa Catarina e São Paulo (Orozco, 2012; Di Iorio, 2013). Existem registros de ataques desta espécie a frutíferas e roseira (Garcia et al., 1993; Garcia \& Corseuil, 1998/1999; Cunha et al., 2007; Aydin, 2011) e também em plantações de milho na fase reprodutiva (Cunha et al., 2007). Micó et al. (2000) descreveram o estágio larval desta espécie a partir do terceiro ínstar. Para G. hebraica existe registro para o Amazonas, Pará, Espírito Santo, Mato Grosso do Sul, Rio de Janeiro e Santa Catarina (Di Iorio, 2013).

Hologymnetis undulata é a única espécie do gênero que se encontra na América do Sul (Ratcliffe \& Deloya, 1992). No Brasil já foi encontrada na região Central, Sudeste e Sul, com registros para o Distrito Federal, Goiás, Mato Grosso, Minas Gerais, Rio de Janeiro, São Paulo, Paraná e Rio Grande do Sul. Não existem registros ou descrições dos estágios imaturos. Adultos se alimentam de flores presentes nas regiões secas como a Caatinga e o Cerrado.

Existem registros das espécies $H$. albiventris e H. brasiliensis para Minas Gerais e de H. liturata para o Mato Grosso (Di Iorio, 2013). O gênero Hoplopygothrix possui apenas duas espécies, $H$. atropurpurea que se encontra na parte Sul, Sudeste e Centro Oeste do Brasil, com registros para Goiás, Minas Gerais, Paraná, Rio de Janeiro, Rio Grande do Sul, Santa Catarina e São Paulo e, H. boliviensis, com apenas um indivíduo (macho) coletado no mês de novembro na Bolívia (Ratcliffe, 2005; 2011). Existe registro de I. bonplandi para o Pará, Minas Gerais e Rio de Janeiro e de M. maculosa para o Brasil (Di Iorio, 2013).

Os Cetoniidae são conhecidos como besouros de flores e frutas, e por voarem a longas distâncias em busca de alimento e de outros recursos (Le Gall, 2010). Podem ser encontrados em flores e frutas maduras, sendo muitas espécies facilmente coletadas usando armadilhas iscadas com frutas fermentadas. Na literatura são encontrados vários trabalhos que utilizam frutas e/ou outros substratos em fermentação para coleta, principalmente banana, abacaxi, caldo de cana, vinho e cerveja (Haddad, 2003; Pacheco et al., 2006; Fierros-López, 2008; Orozco, 2012; Rodrigues et al., 2013b; Touroult \& Le 
Gall, 2013). No entanto, não existe nenhum trabalho que tenha avaliado e comparado a abundância e riqueza de Cetoniidae entre os tipos de iscas. Vários fatores (por exemplo, características da área, velocidade do vento, características da isca) podem interferir na atratividade dos Cetoniidae em função do tipo de isca utilizada. Vale destacar que neste estudo algumas espécies só foram coletadas em um determinado tipo de isca, como $A$. baeri, Gymnetis sp. e H. undulata na isca de banana e as espécies G. rubrocincta e $M$. maculosa na isca de abacaxi.

Foi realizado teste de atratividade de Pachnoda marginata (Drury) para banana e mais 34 compostos sintéticos e foi observado que, banana e 17 compostos principalmente os compostos fenólicos, ésteres de fruta, ácido isovalérico, acetona, e alguns terpenos de frutos e florais, foram significativamente atraentes (Larsson et al., 2003). Dessa forma ressalta-se a importância de uma padronização das armadilhas de coleta bem como das iscas utilizadas para facilidade na comparação dos dados obtidos em diferentes estudos. Por outro lado, o teste de novas frutas, que não são comumente relatadas na literatura, ou até mesmo o teste de frutas nativas e exóticas pode permitir a coleta de espécies que não são atraídas e coletadas com as iscas frequentemente utilizadas.

Estudos sobre fenologia, dinâmica populacional, associações de habitats e uso como indicadores ecológicos em Cetoniidae já foram realizados por Donaldson (1981), Thomas (1993), Morón (1995), Bouyer et al. (2007) entre outros. Apesar da importância de algumas espécies como polinizadores (Singer \& Cocucci, 1997; Micó \& Galante, 1998; Peter \& Johnson, 2009) o papel funcional da maioria das espécies ainda é desconhecido. Na África Ocidental foi encontrada forte relação entre a diversidade de Cetoniidae (2.217 indivíduos e 31 espécies) e o tipo de habitat (a grande maioria das espécies são endêmicas, o que facilita a avaliação assim como a relativa facilidade de identificação e também possuem associação com micro-habitat, como material em decomposição) sugerindo que Cetoniidae pode ser utilizado como indicador quando associado com informações de outros taxa (Touroult \& Le Gall, 2013), entretanto, a baixa diversidade de Cetoniidae encontrada (651 indivíduos e 5 espécies) em Minas Gerais e a falta de informação sobre a ecologia do grupo na região Neotropical não permitem considerá-los como candidatos na avaliação de habitat no Brasil (Puker et al., 2014).

Apesar de o Cerrado apresentar características semelhantes a Savana como plantas endêmicas, microclimas com temperatura e umidade favoráveis e a presença de espécies de Cetoniidae endêmicas (Deschodt et al., 2009; Rerissinotto \& Orozco, 2013), 
a abundância e riqueza de Cetoniidae encontradas neste estudo, bem como nos demais trabalhos realizados no Brasil são muito distintas das encontradas na África Ocidental onde foram coletados 2.217 indivíduos e 31 espécies (Touroult \& Le Gall, 2013).

É importante destacar também que este estudo foi realizado em apenas um tipo de fitofisionomia do Cerrado (cerrado sensu stricto) e que resultados distintos podem ser encontrados se analisados outros tipos de fitofisionomias principalmente devido às diferenças na cobertura vegetal (áreas abertas $\mathrm{x}$ áreas mais fechadas) o que modifica $\mathrm{o}$ sombreamento e a retenção da umidade de cada área.

A abundância de Cetoniidae no Cerrado é maior no período chuvoso, pois é nesse período que existe maior disponibilidade de alimentos. A sazonalidade provoca grande influência na vegetação (Becerra et al., 2009) aumentando ou diminuindo os recursos alimentares, com isso alterando a abundância de insetos (Oliveira \& Frizzas, 2008). Em áreas com uma estação seca bem definida a abundância de insetos tende a ser menor nessa época (Wolda, 1978, Pinheiro et al., 2002). A sazonalidade na abundância de insetos é sincronizada com a disponibilidade de recursos alimentares, e esses por sua vez variam estacionalmente em função do clima (Wolda, 1978; Silva et al., 2011).

As variáveis climáticas temperatura e umidade têm efeito significativo na diversidade de Cetoniidae (tabela 3). A temperatura é uma das variáveis climáticas que mais influenciam a atividade de muitas espécies de insetos, determinando as taxas de desenvolvimento e a atividade reprodutiva dos adultos. A umidade relativa do ar e as chuvas também apresentam forte influência na variação temporal de insetos tropicais, mas muitas vezes não são encontradas correlações diretas com a variação temporal desses organismos ao longo do ano. Contudo, existem evidências de que nos trópicos as primeiras chuvas que ocorrem no final da estação seca funcionam como um mecanismo ou um "gatilho" para a retomada inicial da atividade em insetos (Wolda, 1988).

As espécies residentes no Cerrado, bioma de alta biodiversidade e um dos mais ameaçados do mundo (Mittermeyer et al., 2005), ilustram a possibilidade de conhecer os organismos que o compõem para qualquer política de conservação e destinação do uso da terra. Os resultados obtidos permitem continuar os estudos de levantamento de fauna em outras fitofisionomias por apresentarem recursos alimentares diferenciados, assim como microclimas, podendo existir outras espécies nessas áreas que não foram coletadas no Cerrado stricto sensu. 


\section{CONCLUSÃO}

No Cerrado sensu stricto do Distrito Federal existem, pelo menos, 9 gêneros e 16 espécies de Cetoniidae.

Das espécies coletadas, três ainda não haviam sido registradas para o Cerrado: Allorrhina cornifrons, Gymnetis flavomarginata e Gymnetis rubrocincta.

As espécies mais abundantes foram Gymnetis rufilatris, Gymnetis hebraica e Inca bonplandi.

O maior número de indivíduos e de espécies foi coletado no mês de novembro.

Existe variação temporal para a abundância e riqueza de Cetoniidae, a qual está diretamente ligada à variação temporal do Cerrado com maior número de indivíduos e de espécies no período chuvoso.

As variáveis climáticas temperatura e umidade têm efeito significativo na diversidade de Cetoniidae. 


\section{REFERÊNCIAS}

AGOSTINELLI, C.; LUND, U. R package 'circular': Circular Statistics (version 0.4-7). URL https://r-forge.r-project.org/projects/circular/. 2013.

ANDRESEN, E.; FEER, F. The role of dung beetles as secondary seed dispersers and their effect on plant regeneration in Tropical Rainforests. Seed fate. Predation, dispersal and seedling establishment. 331-349. CABI, Oxon, UK. 2005.

ARNETT Jr, R.H.; THOMAS, M.C.; SKELLEY, P.E.; FRANK, J.H. American beetlesvolume 2. Polyphaga: Scarabaeoidea through Curculionoidea. 54-96. CRC. Boca Raton, FL. 2002.

AYDIN, G. Plant phenology-related shifts in color preferences of Epicometis (Tropinota) hirta (Coleoptera: Scarabaeidae: Cetoniinae) adults - key to effective population monitoring and suppression. Florida Entomologist. 94(4): 832-838. 2011.

BECERRA, J. A. B.; SHIMABUKURO, Y. E.; ALVALÁ, R. C. S. Relação do padrão sazonal da vegetação com precipitação na região de Cerrado da Amazônia legal, usando índices espectrais de vegetação. Revista Brasileira de Meteorologia. 24(2): 125-134. 2009.

BOUYER, J.; SANA, Y.; SAMANDOULGOU, Y. ; CESAR, J.; GUERRINI, L.; KABORE-ZOUNGRANA, C.; DILIEU, D. Identification of ecological indicators for monitoring ecossystem health in the trans-boundary W Regional park: a pilot study. Biological Conservation. 138: 73-88. 2007.

BRANNSTROM, C.; JEPSON, W.; FILIPPI, A. M.; REDO, D.; XU, Z.; GANESH, S. Land change in the Brazilian Savanna (Cerrado), 1986-2002: Comparative analysis and implications for land-use policy. Land Use Policy 25. 579-595. 2008.

CHERMAN, M. A.; MORÓN, M. A. Validación de la família Melolonthidae Leach, 1819 (Coleoptera: Scarabaeoidea). Acta Zoológica Mexicana. 30(1): 201-220. 2014.

COSTA LIMA, A. Insetos do Brasil. 08 Tomo. Coleoptera. 2a Parte. Escola Nacional de Agronomia. Série Didática. 10: 6-8. 1953.

CUNHA, U.S.; GRÜTZMACHER, A. D.; MARTINS, J. F. S.; STEFANELLO, G. J.; JARDIM, E. O. Ocorrência de Euphoria lurida (Fabricius) (Coleoptera: Scarabaeidae) em milho cultivado em Várzea no Rio Grande do Sul. Neotropical Entomology. 36 (6): 976-979. 2007.

DESCHODT, C.M.; SCHOLTZ, C.H.; KRYGER, U. Description of the larva and pupa of Ichnestoma stobbiai (Scarabaeidae: Cetoniinae), a range-restricted species of conservation concern. African Entomology. 17(1): 43-50. 2009.

DI IORIO, O. A review of the Cetoniinae (Coleoptera: Scarabaeidae) from Argentina and adjacent countries: systematics and geographic distributions. Zootaxa. 3668 (1): 001087. 2013. 
DONALDSON, J.M.I. Population dynamics of adult Cetoniinae (Coleoptera: Scarabaeidae) and their relationship to meteorological conditions. Phytophylactica. 13: 11-21. 1981.

FIERROS-LÓPEZ, H. E. Cetoninos dos localidades de Jalisco, México (Coleoptera: Scarabaeidae: Cetoniinae). Dugesiana. 15(2): 147-151. 2008.

GALLO, D.; NAKANO, O.; SILVEIRA NETO, S.; CARVALHO, R.P.L.; BAPTISTA, G.C.; BERTI FILHO, E.; PARRA, J.R.P.; ZUCCHI, R.A.; ALVES, S.B.; VENDRAMIM, J.D.; MARCHINI, L.C.; LOPES J.R.S.; OMOTO, C. Entomologia Agrícola. Piracicaba, Fundação de Estudos Agrários Luiz de Queiroz. 920p. 2002.

GARCIA, A. H.; CUNHA, M. G.; VELESO, V. R. S. Flutuação populacional de Euphoria lurida (Fabricius, 1775) (Coleoptera - Scarabaeidae) em pomar cítrico. Anais Escola de Agronomia e Veteterinária. 23(1): 199-204. 1993.

GARCIA, F. R. M.; CORSEUIL, E. Population fluctuation of cerambycidae and Scarabaeidae (Coleoptera) on peach orchards in Porto Alegre, Rio Grande do Sul. Revista da Faculdade de Zootecnia, Veterinária e Agronomia Uruguaiana. 5/6(1): 69-81. 1998/1999.

GARCIA, F.P., RODRIGUES, S.R., BAGNARA, C.A.C. \& OLIVEIRA, D.S. Survey of saproxylophagous Melolonthidae (Coleoptera) and some biological aspects in Aquidauana, MS. Biota Neotropica. 13(3): 000-000. 2013.

GONÇALVES, T. T.; LOUZADA, J. N. C. Estratificação vertical de coleópteros carpófilos (Insecta: Coleoptera) em fragmentos florestais do sul do Estado de Minas Gerais, Brasil. Ecologia Austral. 15: 101-110. 2005.

HADDAD, C.R. Fruit chafers (Coleoptera: Scarabaeidae: Cetoniini) of the Ndumo Game Reserve and Tembe Elephant Park, KwaZulu-Natal. African Entomology. 11(1): 130133. 2003.

HALFFTER, G.; FAVILA, M. E. The Scarabaeinae an animal group for analysing, inventorying and monitoring biodiversity in Tropical Rainforest and modified landscapes. Biology International. 27: 15-21. 1993.

HOLM, E.; MARAIS, E. Fruit chafer of Southern Africa (Scarabaeidae: Cetoniini). Ekogilde, Hartebeespoort, South Africa. :1-326. 1992.

JÁKL, S. Results of entomological expeditions to Yamdena, Larat, Tandula, Selaru and Molu islands (Indonesia, Moluccas, Tanimbar islands) with the description of new genus, three new species and four new subspecies (Coleoptera: Cetoniinae). Studies and reports of District Museum Prague-East. Taxonomical Series. 5 (1-2): 139-158. 2009.

JOST, L. Entropy and diversity. Oikos. 113:2. 2006.

KOVACH, W. L. Oriana - Circular Statistics for Windows, ver. 4. Kovach Computing Services, Pentraeth, Wales, U. K. 2011. 
KRELL, F. T.; SIMON, U.; ZOTZ, G. Cetoniinae developing in a living stalk of Bromeliaceae (Coleoptera:Scarabaeidae: Cetoniinae: Gymnetini). The Coleopterists Bulletin. 56(4): 533-539. 2002.

KRIKKEN, J. A new key to the suprageneric taxa in the beetle family Cetoniidae, with annotated lists of the known genera. Zoologische Verhandelingen. Leiden. 210: 1-175. 1984a.

KRIKKEN, J. A generic reclassification of the Afrotropical Bolboceratini (Coleoptera: Geotrupidae). Zoologische Mededelingen. 58(3): 23-45. $1984 \mathrm{~b}$.

KUMBHAR, S.M.; MAMLAYA, A.B.; PATIL, S.J.; BHAWANE, G.P. Biology of Chiloloba orientalis. University of Wisconsin Library. 2012.

LARSSON, M.C.; STENSMYR, M. C.; BICE, S. B.; HANSSON, B. S. Attractiveness of fruit and flower odorants detected by olfactory receptor neurons in the fruit chafer Pachnoda marginata. Journal of Chemical Ecology. 29(5). 2003.

LE GALL, P. Affinités biogéographiques des Insectes du "Dahomey gap" présence d'une popuiation de Goliathus goliatus Linné, 1771, au Bénin (Coleoptera, Scarabaeidae, Cetoniinae). Bulletin de la Société entomologique de France. 115(1): 17-21. 2010.

LUÇARDO, M.; OLIVEIRA, C.M.; FRIZZAS, M.R. Scarabaeoidea (Insecta: Coleoptera) no Cerrado brasileiro: estado atual do conhecimento. Ciência Rural. 44(4): 652-659. 2014.

MENDOZA, C. A theorem for Rayleigh's method of dimensional analysis and its proof. Mechanics Research Communications. 21(2): 103-107. 1994.

MICÓ, E.; GALANTE, E. The behavior of Aethiessa floralis (Fabricius, 1787) (Coleoptera: Scarabaeoidea: Cetoniidae) visiting Onopordum macracanthum Schousboe (Compositae). Elytron. 12: 69-76. 1998.

MICÓ, E.; GALANTE, E. Biology and new larval descriptions for three cetoniine beetles (Coleoptera: Scarabaeidae: Cetoniinae: Cetoniini: Cetoniina, Leucocelina). Annals of the Entomological Society of America. 96(2): 95-106. 2003.

MICÓ, E.; HALL, W.E.; RATCLIFFE, B.C. Descriptions of the larvae of Hoplopyga singularis (Gory and Percheron) and Hologymnetis cinerea (Gory and Percheron) with a revised key to the larvae of New World Gymnetini (Coleoptera: Scarabaeidae: Cetoniinae). The Coleopterists Bulletin. 55: 205-217. 2001.

MICÓ, E.; MORÓN, M. A.; ŠÍPEK, P.; GALANTE, E. Larval morphology enhances phylogenetic reconstruction in Cetoniidae (Coleoptera: Scarabaeoidea) and allows the interpretation of the evolution of larval feeding habits. Systematic Entomology. 33: 128144. 2008.

MICÓ, E.; SMITH, A.; MORÓN, M.A. New larval descriptions for two species of Euphoria Burmeister (Coleoptera: Scarabaeidae: Cetoniinae: Cetoniini: Euphoriina) with a key to the known larvae and a review of the larval biology for species in the genus. Systematics Research Collections, University of Nebraska State Museum. 2000. 
MITTERMEIER, R. A.; GIL, P. R.; HOFFMANN, M.; PILGRIM, J.; BROOKS, T.; MITTERMEIER, C. G.; LAMOREUX, J.; FONSECA, G. A. B. Hotspots revisited: Earth's biologically richest and most threatened terrestrial ecoregions. Mexico, CEMEX. 392p. 2005.

MOLINA, J.M. Incidencia de Tropinota squalida (Scopoli, 1783) (Coleoptera: Scarabaeidae) en el cultivo del arándano en Huelva (España): problemática asociada a su control. Zapateri: Revista Aragonesa de Entomología. 9: 93-98. 2001.

MORÓN, M.A. Fenology and habits of the Cetoniinae (Coleoptera: Melolonthidae) in the Xalapa-Coatepec area, Veracruz, Mexico. Giornale Italiano di Entomologia. 7: 317332. 1995.

MORÓN, M.A. Diversidad y distribución del complejo gallina ciega (Coleoptera: Scarabaeoidea). In: Rodríguez del Bosque L. A.; Morón, M.A. Plagas del Suelo. 41-64. 2010.

MYERS, N.; MITTERMEIER, R. A.; MITTERMEIER, C. G.; FONSECA, G. A. B.; KENT, J. Biodiversity hotspots for conservation priorities. Nature. 403: 853 - 858. 2000.

OLIVEIRA, C. M.; FRIZZAS, M. R. Insetos de Cerrado: distribuição estacional e abundância. Boletim de pesquisa e desenvolvimento. Embrapa Cerrados. 26p. 2008.

OLIVEIRA, C. M.; FRIZZAS, M. R. Field biology of the beetle Aegopsis bolboceridus in Brazil, with a list of host plant. Journal of Insect Science. 13: 48. 2013.

OROZCO, J. Monographic revision of the American genus Euphoria Burmeister, 1842 (Coleoptera: Scarabaeidae: Cetoniinae). The Coleopterists Bulletin. 11:1-182. 2012.

OROZCO, J.; PARDO-LOCARNO, L.C. Description of immature stages of three species of American Cetoniinae (Coleoptera: Scarabaeidae: Cetoniinae). Zootaxa. 769: 1-14. 2004.

PACHECO, F. C.; DELOYA, C.; CORTES G. P. Phytophagous scarab beetles from the Central Region of Guerrero, Mexico (Coleoptera: Scarabaeidae: Melolonthinae, Rutelinae, Dynastinae, Cetoniinae). Revista Colombiana de Entomología. 32(2). 2006.

PEJAVER, M. K.; GUJARATHI, G. R. Conserving through composting- the lifecycle study of pest turned partner. National Conference on Biodiversity: status and challenges in Conservation - 'FAVEO'. 2013.

PERISSINOTTO, R.; OROZCO, J. Eudicella trimeni Janson, 1884 (Coleoptera: Scarabaeidae: Cetoniinae: Goliathini): description of larva with notes on conservation status, biology and taxonomy. African Invertebrates. 54(2): 417 - 426. 2013.

PETER, C.I.; JOHNSON, S.D. Pollination by flower chafer beetles in Eulophia ensata and Eulophia welwitschii (Orchidaceae). South African Journal of Botany. 75: 762-770. 2009. 
PINHEIRO, F.; DINIZ, I. R.; COELHO, D.; BANDEIRA, M. P. S. Seasonal pattern of insect abundance in the Brazilian Cerrado. Austral Ecology (Print), Austrália. 27(2): 132136. 2002.

PUKER, A.; AD’VINCULA, H. L.; KORASAKI, V.; FERREIRA, F. N. F.; OROZCO, J. Biodiversity of Cetoniinae beetles (Coleoptera: Scarabaeidae) in introduced and native habitats in the Brazilian Atlantic Forest. Entomological Science. 17: 309-315. 2014.

PUKER, A.; LOPES-ANDRADE, C.; ROSA, C.S.; GROSSI, P.C. New records of termite hosts for two species of Hoplopyga, with notes on the life cycle of Hoplopyga brasiliensis (Coleoptera: Scarabaeidae: Cetoniinae). Annals of the Entomological Society of America. 105(6): 872-878. 2012.

R Core Team. R: A language and environment for statistical computing. R Foundation for Statistical Computing, Vienna, Austria. URL http://www.R-project.org/ . 2014.

RATCLIFFE, B.C. A checklist of the Scarabaeoidea (Coleoptera) of Panama. Zootaxa. 32: 1-48. 2002.

RATCLIFFE, B.C. Lectotype designations in the New World Gymnetini (Coleoptera: Scarabaeidae: Cetoniinae). Zootaxa. 729: 1-19. 2004.

RATCLIFFE, B.C. A Review of the South American Genus Hoplopygothrix Schürhoff (Coleoptera: Scarabaeidae: Cetoniinae: Gymnetini). The Coleopterists Bulletin. 59(1): 136-142. 2005.

RATCLIFFE, B.C. A review of the Blaesiina (Coleoptera, Scarabaeidae, Cetoniinae, Gymnetini). ZooKeys. 34: 105-128. 2010.

RATCLIFFE, B.C. Hoplopygothrix Schürhoff (Coleoptera: Scarabaeidae: Cetoniinae: Gymnetini) Revisited: A new species and country record for Bolivia. The Coleopterists Bulletin. 65(1): 63-66. 2011.

RATCLIFFE, B.C. A Revision of the Neotropical Genus Allorrhina Burmeister, 1842 (Coleoptera: Scarabaeidae: Cetoniinae: Gymnetini). The Coleopterists Bulletin. 69(1):91113. 2015.

RATCLIFFE, B.C.; DELOYA, A. C. The Biogeography and Phylogeny of Hologymnetis (Coleoptera: Scarabaeidae: Cetoniinae) with revision of the genus. The Coleopterists Bulletin, 46(2): 16 1-202. 1992.

RATCLIFFE, B.C.; JAMESON, L. M. The revised classification for Scarabaeoidea: what the hell is going on?. Museum, University of Nebraska. State Papers in Entomology, University of Nebraska - Lincoln. 2004.

RATCLIFFE, B.C.; MICÓ, E. A Review of the Neotropical genus Neocorvicoana Ratcliffe and Micó, New Genus (Coleoptera: Scarabaeidae: Cetoniinae: Gymnetini). The Coleopterists Bulletin. 55(3): 279-296. 2001.

RATCLIFFE, B.C.; PAULSEN, M.J. The Scarabaeoid Beetles of Nebraska (Coleoptera: Scarabaeoidea). University of Nebraska State Museum Bulletin. 22: 1-570. 2008. 
RITCHER, P. O. Biology of Scarabaeidae. Annual Review of Entomology. 3: 311-334. 1958.

RODRIGUES, M.M.; UCHOA, M.A.; IDE, S. Dung beetles (Coleoptera: Scarabaeoidea) in three landscapes in Mato Grosso do Sul, Brazil. Brazilian Journal of Biology. 73: 1. 2013a.

RODRIGUES, S.R.; OLIVEIRA. J.L.N.; BAGNARA, C.A.C.; PUKER, A. Cetoniinae (Coleoptera: Scarabaeidae) attracted to fruit-baited traps near Aquidauana, Mato Grosso Do Sul, Brazil. The Coleopterists Bulletin. 67(2): 119-122. 2013b.

SÁNCHEZ-GALVÁN, I. R.; QUINTO, J.; MICÓ, E.; GALANTE, E.; MARCOSGARCÍA, M. A. Facilitation among saproxylic insects inhabiting tree hollows in a Mediterranean Forest: The Case of Cetonids (Coleoptera:Cetoniidae) and Syrphids (Diptera: Syrphidae). Environmental Entomology. 43(2): 336-343. 2014.

SANO, E. E.; ROSA, R.; BRITO, J. L. S.; FERREIRA, L. G. Land cover mapping of the tropical savanna region in Brazil. Environmental Monitoring and Assessment. 166:113124. 2010.

SEYMOUR, R.S.; WHITE, C.R.; GIBERNAU, M. Environmental biology: heat reward for insect pollinators. Nature. 426: 6964. 2003.

SHUTTLEWORTH, A.; JOHNSON, S. D. New records of insect pollinators for South African asclepiads (Apocynaceae: Asclepiadoideae). South African Journal of Botany. 75: 689-698. 2009.

SILVA, C. R.; SOUZA, K. B.; FURTADO, W. F. Evaluation of the progress of intensive agriculture in the Cerrado Piauiense - Brazil. IERI Procedia. 5: 51 - 58. 2013.

SILVA, N.A.P., FRIZZAS, M.R., OLIVEIRA, C.M. Seasonality in insect abundance in the Cerrado of Goiás State, Brazil. Revista Brasileira de Entomologia. 55(1): 79-87. 2011.

SINGER, R.B.; COCUCCI, A.A. Pollination of Pteroglossaspis ruwenzoriensis (Rendle) Rolfe (Orchidaceae) by beetles in Argentina. Botanica Acta. 110: 338-342. 1997.

ŠÍPEK, P.; KRÁL, D. Immature stages of the rose chafers (Coleoptera: Scarabaeidae: Cetoniinae): a historical overview. Zootaxa. 3323: 1-26. 2012.

SUBCHEV, M.A.; TOSHOVA, T. B.; ANDREEV, R. A.; PETROVA, V. D.; MANEVA, V. D.; SPASOVA, T. S.; MARINOVA, N. T.; MINKOV, P. M.; VELCHEV, D. I. Employing floral baited traps for detection and seasonal monitoring of Tropinota (Epicometis) hirta (Poda) (Coleoptera: Cetoniidae) in Bulgaria. Acta Zoologica Bulgarica. 63(3): 269-276. 2011.

THOMAS, D.B. Scarabaeidae (Coleoptera) of the Chiapanecan forests: a faunal survey and chorographic analysis. The Coleopterists Bulletin. 47: 363-408. 1993.

TOUROULT, J.; LE GALL, P. Fruit feeding Cetoniinae community structure in an anthropogenic landscape in West Africa. Journal of Insect Conservation. 17: 23-34. 2013. 
VANIN, S.A.; COSTA, C. Larvae of Neotropical Coleoptera. IX: Scarabaeidae, Cetoniinae, Gymnetini. Revista Brasileira de Entomologia. 28(3): 329-335. 1984.

VAZ DE MELLO, F.Z. Estado atual de conhecimento dos Scarabaeidae S. STR. (Coleoptera: Scarabaeoidea) do Brasil. Monografías Tercer Milenio. 1: 183-195. 2000.

VAZ DE MELLO, F.Z.; LOUZADA, J.N.C.; SCHOEREDER. J.H. New Data and Comments on Scarabaeidae (Coleoptera: Scarabaeoidea) associated with Attini (Hymenoptera: Formicidae). The Coleopterists Bulletin. 52(3): 209-216. 1998.

VUTS, J.; ZOLTÁN, I.; TÓTH, M. Development of an attractant-baited trap for Oxythyrea funesta Poda (Coleoptera: Scarabaeidae, Cetoniinae). Zeitschrift für Naturforschung C. 63(9-10): 761-8. 2008.

VUTS, J.; SZARUKÁN, I.; SUBCHEV, M.; TOSHOVA, T.; TÓTH, M. Improving the xoral attractant to lure Epicometis hirta Poda (Coleoptera: Scarabaeidae, Cetoniinae. Journal of Pest Science. 83:15-20. 2010.

WOLDA, H. Seasonal fluctuations in rainfall, food and abundance of tropical insects. The Journal of Animal Ecology. 47(2): 369-381. 1978.

WOLDA, H. Insect seasonality: why? Annual Review of Ecology and Systematics. 19: 1-18. 1988. 